\title{
Modeling and Error Analysis of the Parallelism Measurement for Linear Rolling Guide Pair
}

\author{
Yi Liang, Yulin Wang, Aihua Yin, Li Zu, and Dan Xu
}

\begin{abstract}
The parallelism of a linear rolling guide pair directly affects the machining precision of machine tools. Therefore, it's necessary and meaningful to find the measurement method to test them. A test method of parallelism of rolling linear guide pairs based on non-contact laser displacement sensors has been put forward. The paper derives an analytical testing algorithm of measurements of five laser displacement sensors to obtain the parallelism and their error analysis of linear rolling guide pairs. The parallelism of linear guide pairs includes five movement precisions which are horizontal parallelism, vertical parallelism, elevation angle, deflection angle and roll angle of a slider block relative to its guide way. The converting formulas from the laser sensors' measurement to the two parallelisms and three angles are derived based on the basic theory of three-points can determine a plane. Because the sensors are installed with inevitably errors, the deviation of two parallelisms and three angles caused by the errors are analyzed. Results show that two parallelisms and three rotation angles depend critically on the measuring length of five laser sensors. The paper provides an effective method for measuring the parallelism and makes progress of the automatic detection of a linear rolling guide pair.
\end{abstract}

Index Terms-Error analysis, Linear roller guide, movement precision, parallelism.

\section{INTRODUCTION}

The rolling linear guide pairs are widely used in automation equipments and high-end CNC tools as precision guide members, because of its high linear positioning accuracy. The parallelism, namely straightness, of guide pairs in translation motion is a significant index of accessing accuracy, since which directly affects their own service life and the machining precision of machine tools. Therefore, it's necessary and meaningful to test and evaluate the parallelism of guide pairs.

In recent years, a few of researchers and engineers had studied on how to test the precision of linear guide pairs. Liu and C. H. Liu used optical measurement technology to measure the guide straightness by an intuitive and reliable method [1], [2]. Based on relativity measure theory, Li Wei designed and manufactured three automatic measuring instruments by applying geometrical simulation and automatic measuring technique. The measure result indicated that the instrument advanced the measuring veracity and efficiency [3]. L. J. Zhu and C. D. Hu introduced some method of measuring guide straightness error [4], [5]. Y. Liu

Manuscript received December 3, 2014; revised June 20, 2015. This work was sponsored so honorably by the National Natural Science Foundation of China (Youth Science Fund 51405233) and National Science and Technology Major Project of China (2012ZX04002021).

The authors are with Nanjing University of Science and Technology, 210094, P.R.China (e-mail: liangyi@njust.edu.cn,wyl_sjtu@126.com). used two total stations cooperating with each other to measure the straightness of the guide rail [6]. P. Majda proposed a method of analytical examination of the influence of geometric errors in linear guide way on joint kinematic errors [7]. Yang Ye and Feng used a gantry with five non-contact probe to measure the accuracy of guide way, it has high detection accuracy and it is suitable for different specifications of guide rail [8]. J. Xiong introduced the dynamic measurement of friction and put forward two methods to measure and check motion accuracy [9]. J. L. Sun and M. H. Qian discussed the processing course in quest of linear error by GA [10]. Tao, Yin, Cai and Zhang introduced a system for testing linear motion guide. In the system, laser, optical fiber, displacement sensor, laser collimation technique, force sensor and computers were used to measure and process data. It solved the problem of measuring linear motion guide [11]. Zhong, Tao and Han studied the modeling and test method on the motion precision loss process under load condition [12]. Qing and Hong adopted position sensitive detector (PSD) and laser collimation technique to measure four-degree-of-freedom of linear guide rails, and BP neural network algorithm is used to correct the nonlinearity between actual and ideal output of PSD. The results showed that the four-degree of linear guide rails can be measured with high accuracy [13]. These scholars did many useful and practical works in testing the accuracy of linear guide pairs.

\section{The Test Method of PARALLELISM IN LinEAR Rolling GUIDE PAIRS}

Movement precision refers to the difference between the real and the theoretical displacements of a specific point. According to Mechanical Industry Standards of China JB/T 175.4-2006, the acceptance specifications for the precision test of rolling linear guide includes two aspects, as shown in Fig. 1.

1) The parallelism between the slider movement and the guide bottom reference A, namely vertical parallelism.

2) The parallelism between the slider movement and the guide side reference B, namely horizontal parallelism.

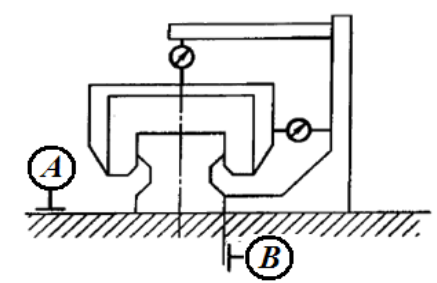

Fig. 1. The precision test of rolling linear guide.

When the guide works in normal, in theory, it could only be 
allowed to move in reciprocating linear motion along the rail direction. Actually, the movement of rolling elements in linear guide pairs is complex. The movement precision is affected by many factors, the manufacture precision of the parts, installation accuracy, preload force, frictional force etc. Therefore, in addition to the moving direction, there are five kind of errors in linear guide pairs, which are the parallelism accuracy (horizontal and vertical) and the accuracy of rotation around the three axes (elevation angle, deflection angle, roll angle). Though there are no standard of the accuracy of rotation in $\mathrm{JB} / \mathrm{T}$ 175.4-2006, we insist test the angles to research linear guide slider's position and orientation because they would influence the machining accuracy.

A coordinate system is established in Fig. 2. Point $O$ is the ideal position of the block on the linear guide, $O^{\prime}$ the actual position. So the translation displacement along to the $X$ and $Y$ direction refer to the horizontal and vertical parallelism of the linear guide pair, respectively. The angles of rotation around three axes, $X, Y$ and $Z$, are the pitch angle $\theta_{x}$, deflection angle $\theta_{y}$ and roll angle $\theta_{z}$, respectively.

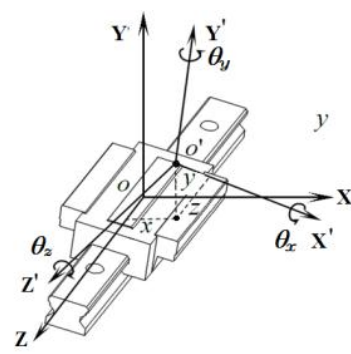

Fig. 2. The coordinate system of a rolling linear guide pair.

In Fig. 3, we designed an instrumentation to measure the horizontal and vertical parallelism and three angle errors of the linear guide pair.

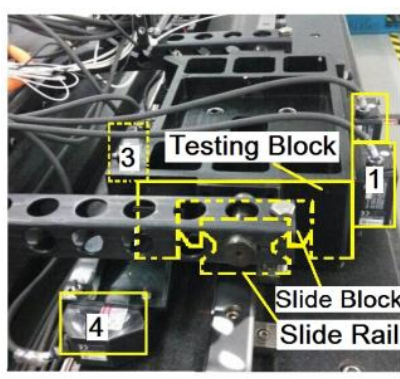

(a)

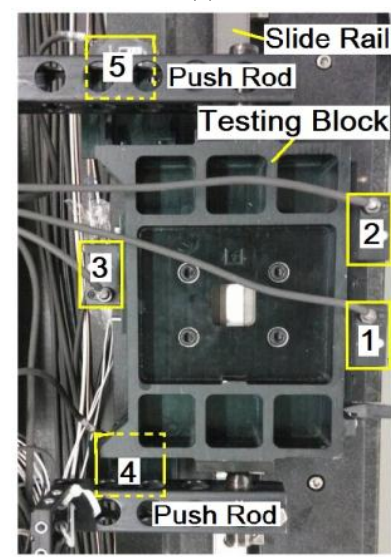

(c)

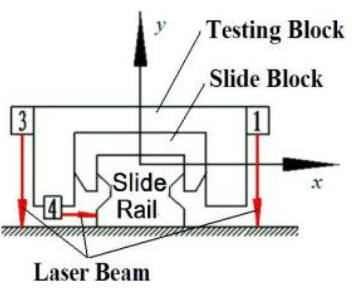

(b)

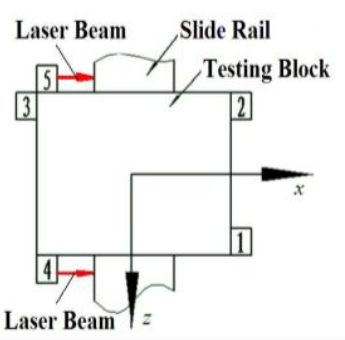

(d)
Fig. 3. Structure of test system.
Fig. 3(a) and Fig. 3(b) show the front view of measuring device, a practical photograph and schematic diagram respectively. Fig. 3(c) and Fig. 3(d) are the top view of them. Two push rods in Fig. 3(c) may push the testing block and slide block move along the rail back and forth, when testing will be carried out. In the Fig. 3, 1 to 5, five non-contact laser displacement sensors, are installed on the testing block, which is mounted on the slide block to be measured. The laser light of the sensors 1, 2 and 3 beam down on the granitic surface which is a reference plane. The laser light of the sensors 3 and 4 beam on one side of guide. When the slider is driven to move along the guide rail, the laser displacement sensors 1-5 would test the displacement variation $\left(x_{1}, x_{2}, x_{3}, x_{4}, x_{5}\right.$ ). Subsequently, the measured result would be saved and analyzed by the dynamic measurement software to calculate parallelisms and angle errors.

\section{ThE DERIVATION OF PARALLELISM OF ROLLING LINEAR GUIDE PAIRS}

\section{A. The Angle of Rotation}

Because the five laser sensors are perpendicular to their relevant test reference surfaces, the angles of slider block can be calculated by the measurement of sensors. So the pitch angle $\theta_{x}$ of the slider around the $X$ direction is obtained by the measurement of sensors 1 and 2. The deflection angle $\theta_{y}$ around the $Y$ direction can be calculated by the measurement results of sensors 4 and 5 . The roll angle $\theta_{z}$ around the $Z$ direction can be measured with sensors 2 and 3 . The geometrical relationship and measuring principle are shown in Fig. 4.

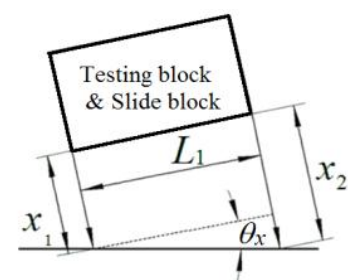

Fig. 4. Measuring principle of slider angle.

By the geometric relationships, the formulas of rotation are

$$
\left\{\begin{array}{l}
\theta_{x}=\arctan \left(\frac{x_{1}-x_{2}}{L_{1}}\right) \\
\theta_{y}=\arctan \left(\frac{x_{2}-x_{3}}{L_{2}}\right) \\
\theta_{z}=\arctan \left(\frac{x_{4}-x_{5}}{L_{3}}\right)
\end{array}\right.
$$

where

$\theta_{x}, \theta_{y}, \theta_{z}$-The angles of slider around $X, Y, Z$ axis;

$x_{1}, x_{2}, x_{3}, x_{4}, x_{5}$-The measurements by five laser sensors;

$L_{1}$-The distance between the light spot of sensors 1 and 2;

$L_{2}$-The distance between the light spot of sensors 2 and 3;

$L_{3}$-The distance between the light spot of sensors 4 and 5 . 


\section{B. The Horizontal Parallelism along the X Direction}

According to Mechanical Industry Standards of China JB/T 175.4-2006, the horizontal parallelism $x$ refers to variation of the distance between the center point of the side surface on the slide block and the side reference plane. However the laser distance sensors 4 and 5 on the front and back of the slide block, which is not the center point. So we need to find a method to convert the measurement of sensors to the horizontal of parallelism $x$ on the center point.

The slider is regarded as a rigid body. Sensors 4 and 5 are symmetrically arranged, by calculating the distance of the slider side midpoint in the $X$ direction. The horizontal parallelism $x$ can be obtained by $x_{4}, x_{5}$ and $h$ (see Fig. 5).

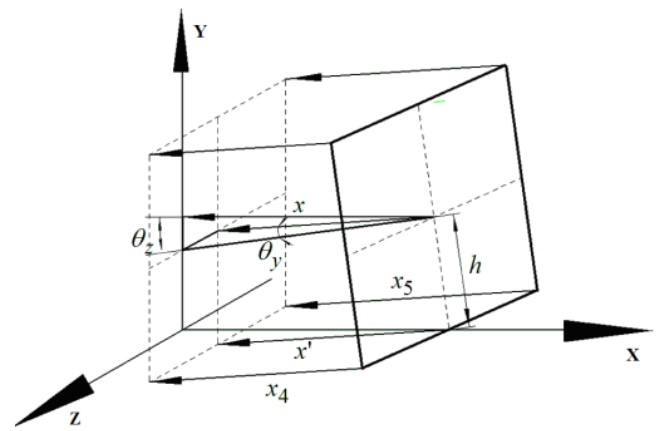

Fig. 5. The measuring principle of the horizontal parallelism.

Since a plane cannot be determined by two sensors, the compensation parameter $h$ is added, which is the vertical distance from the center of slider side to the sensor mounting plane. The perpendicular distance $x$ can be developed to

$$
\begin{aligned}
x & =x^{\prime} \cos \theta_{x} \cos \theta_{z}-h \sin \theta_{z} \\
& =\frac{1}{2}\left(x_{4}+x_{5}\right) \cos \theta_{x} \cos \theta_{z}-h \sin \theta_{z}
\end{aligned}
$$

\section{The Vertical Parallelism along the Y Direction}

Likewise, we need to derive the formula to convert the measurement of sensors 1,2 and 3 to the vertical of parallelism $y$ on the center point of the upper plane of the slider block. The three points of laser sensors can determine the upper plane, shown in Fig. 6. Since the sensors 1, 2 and sensors 2, 3 are installed symmetrically relative to the center plane of the slider, the vertical parallelism $y$ of the slider surface center along the $Y$ direction is equal to the moving distance in the $Y$ direction of the upper plane center.

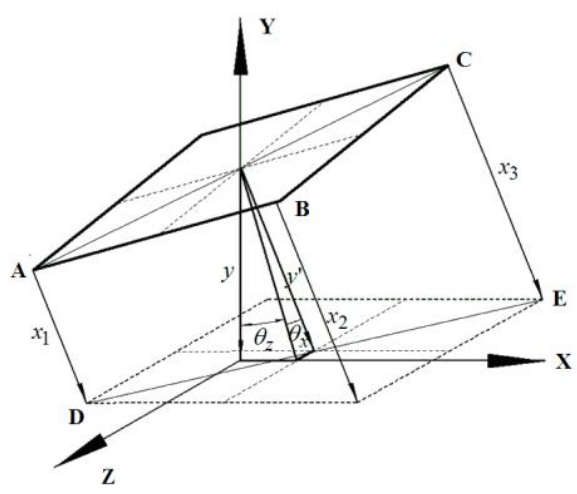

Fig. 6. The measuring principle of the vertical parallelism.
As shown in the Fig. 6, the trapezoid ADEC is formed in the space. Since the angle $\theta_{y}$ about the $Y$-axis does not affect the magnitude of $y^{\prime}$, it can be set to zero. So the perpendicular distance y can be developed to

$$
y=y^{\prime} \cos \theta_{x} \cos \theta=\frac{1}{2}\left(x_{1}+x_{3}\right) \cos \theta_{x} \cos \theta_{z}
$$

\section{THE EFFECT OF INSTALLATION ERRORS ON THE PARALLELISM}

The sensors are mounted on the testing block with inevitably errors. Now we will analyze the deviation of the angle of rotation $\theta_{x}, \theta_{y}, \theta_{z}$ and linear parallelism $x$ and $y$ caused by the installation errors.

\section{A. The Effect of Installation Errors on the Angle of Rotation}

In Fig. 4, it's apparent that the angle of rotation $\theta_{x}$ depends on $x_{1}, x_{2}$ and $L_{1}$. If the actual length of these three parameters are not the exactly same with their ideal value, which is common in engineering field, the angle $\theta_{x}$ will be different from the value we expected. We denote the difference between ideal and actual value as $\Delta x_{1}, \Delta x_{2}$ and $\Delta L_{1}$, respectively. The deviation of the angle of rotation $\theta_{x}$ cause by them is named denoted by $\Delta \theta_{x}$. We use the below formula to analyze the relationship of them.

$$
\Delta \theta_{x}=\frac{\partial \theta_{x}}{\partial x_{1}} \Delta x_{1}+\frac{\partial \theta_{x}}{\partial x_{2}} \Delta x_{2}+\frac{\partial \theta_{x}}{\partial L_{1}} \Delta L_{1}
$$

In which, $\theta_{x}$ can be substituted by the formula (1).

In order to discovery the directive relation of $\Delta L_{1}$ and $\Delta \theta_{x}$, shown in Fig. 7, the other factors $\Delta x_{1}, \Delta x_{2}$ will be fixed unchanged. Fig. 7 shows that the deviation of $\Delta \theta_{x}$ is positively correlated to $\Delta L_{1}$. Furthermore, if the length $L_{1}$ gets smaller, namely, the sensors 1 and 2 are mounted closer, $\Delta L_{1}$ has more influence on $\Delta \theta_{x}$. But even so, the deviation of $\Delta \theta_{x}$ is tiny to $0.00025^{\circ}$ when $\Delta L_{1}$ has a large error of $5 \mathrm{~mm}$. So the error of $\Delta L_{1}$ would not be a critical factor to $\Delta \theta_{x}$.

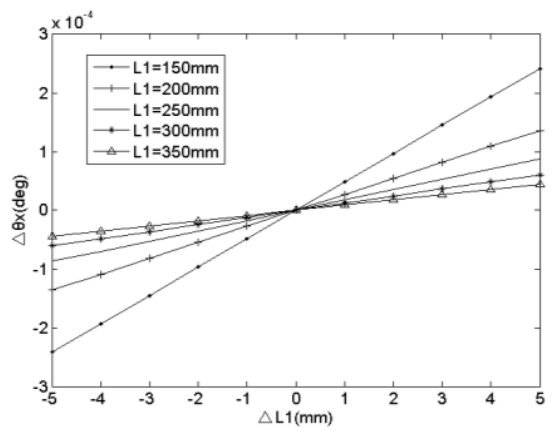

Fig. 7. The effect of error $\Delta L_{1}$ on the angle $\Delta \theta_{x}$.

Similarly, when we analyze the influence of $\Delta x_{1}, \Delta x_{2}$ and $\Delta L_{1}$ will be unchanged. Furthermore, because the reference measurement length $x_{1}$ of laser sensor 1 is $20 \mathrm{~mm}$ and the measuring range of laser sensor 1 is $2 \mathrm{~mm}$, the actual measurement length $x_{1}$ would vary from $18 \mathrm{~mm}$ to $22 \mathrm{~mm}$. When it changes in this range, the variation curves of $\Delta \theta_{x}$ are 
very close to each other and no obvious difference. So, the other curves except $x_{1}=20 \mathrm{~mm}$ are left out and are not shown in Fig. 8. It shows that the deviation of $\Delta \theta_{x}$ is positively correlated to $\Delta x_{1}$. Because the measuring range of laser sensor 1 is $2 \mathrm{~mm}$, the installation error $\Delta x_{1}$ is selected to $-2 \mathrm{~mm}$ to $2 \mathrm{~mm}$. Fig. 8 shows that the $\Delta x_{1}$ has a strong influence on $\Delta \theta_{x}$. When the error $\Delta x_{1}$ is $2 \mathrm{~mm}, \Delta \theta_{x}$ would have a relative huge deviation of $0.45^{\circ}$. So accuracy of the length $x_{1}$ is the key factor guaranteeing precision of the pitch angel $\theta_{x}$.

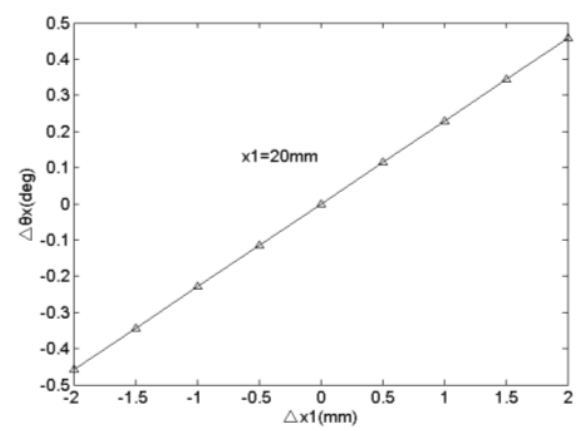

Fig. 8. The effect of error $\Delta x_{1}$ on the angle $\Delta \theta_{x}$.

In formula (1), we know that $x_{2}$ plays the same role with that of $x_{1}$ in determining the pitch angel $\theta_{x}$. We have no use for drawing the variation curves of $\Delta \theta_{x}$ and $\Delta x_{2}$.

From formula (1), it can be seen that $\theta_{y}, \theta_{z}$ are almost the same to $\theta_{x}$. So we needn't to analyze them repeatedly.

\section{B. The Effect of Installation Errors on the Linear Parallelism}

By comparing formulas (2) and (3), we find they are similar to each other. We choose a linear parallelism $y$ to analyze. Observing formulas (1) and (3), we notice that parallelism $y$ is a function of 7 variables. Similar to formula (4), we get the deviation of vertical parallelism $\Delta y$.

$$
\begin{aligned}
\Delta y & =\frac{\partial y}{\partial x_{1}} \Delta x_{1}+\frac{\partial y}{\partial x_{2}} \Delta x_{2}+\frac{\partial y}{\partial x_{3}} \Delta x_{3}+\frac{\partial y}{\partial x_{4}} \Delta x_{4} \\
& +\frac{\partial y}{\partial x_{5}} \Delta x_{5}+\frac{\partial y}{\partial L_{1}} \Delta L_{1}+\frac{\partial y}{\partial L_{3}} \Delta L_{3}
\end{aligned}
$$

To simplify the question, three typical factors $x_{1}, x_{4}, L_{1}$ are chose to be analyzed. Fig. 9 shows that the deviation of $\Delta y$ is positively correlated to $\Delta x_{1}$. When the installation error $\Delta x_{1}$ changes from $-2 \mathrm{~mm}$ to $2 \mathrm{~mm}, \Delta y$ varies from $-1 \mathrm{~mm}$ to $1 \mathrm{~mm}$. It means that $\Delta x_{1}$ has a great effect on $\Delta y$. The accuracy of the length $x_{1}$ is a key factor guaranteeing precision of vertical parallelism $y$.

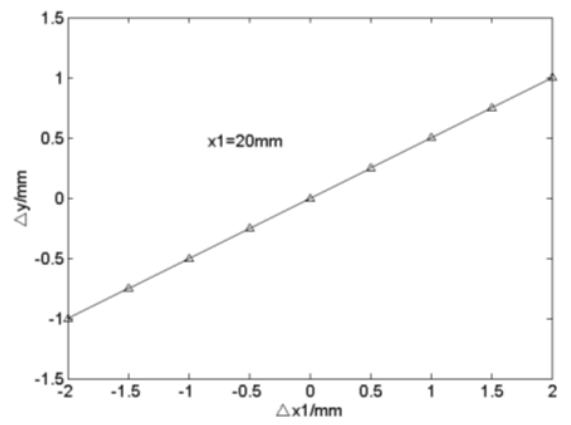

Fig. 9. The effect of error $\Delta x_{1}$ on the parallelism $\Delta y$.
In Fig. 10, the installation error of the laser sensor 4 is analyzed. Because the reference measurement length $x_{4}$ of laser sensor 4 is $50 \mathrm{~mm}$, the actual measurement length $x_{4}$ would vary from $48 \mathrm{~mm}$ to $52 \mathrm{~mm}$. So, there are five curves in the figure. Fig. 10 shows that the deviation of $\Delta y$ is proportional to $\Delta x_{4}$ when $x_{4}=48,49,50 \mathrm{~mm}$ and inversely proportional to $\Delta x_{4}$ when $x_{4}=51,52 \mathrm{~mm}$. However, if we focus on the absolute value of $\Delta y$, we find that $|\Delta y|$ is positively correlated to $\Delta x_{4}$. As for the curve of $x_{4}=48$ or $50 \mathrm{~mm}$, when the installation error $\Delta x_{4}$ changes from $-5 \mathrm{~mm}$ to $5 \mathrm{~mm}, \Delta y$ varies from $-0.0022 \mathrm{~mm}$ to $0.0022 \mathrm{~mm}$. It means that $\Delta x_{4}$ has little effect on $\Delta y$. The accuracy of the length $x_{4}$ is not a critical factor to $y$.

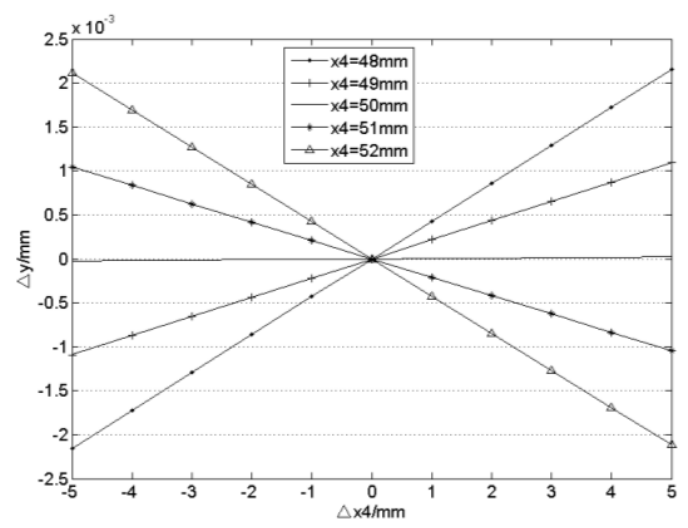

Fig. 10. The effect of error $\Delta x_{4}$ on the parallelism $\Delta y$.

In Fig. 11, the deviation of $\Delta y$ is positively correlated to $\Delta L_{3}$. Furthermore, if the length $L_{3}$ gets smaller, namely, the sensors 4 and 5 are mounted closer, $\Delta L_{3}$ has more influence on $\Delta y$. But even so, the deviation of $\Delta y$ is tiny, $6 \times 10^{-6} \mu \mathrm{m}$ when $\Delta L_{3}$ has a large error of $5 \mathrm{~mm}$. So the error of $\Delta L_{3}$ would not be a critical factor to $\Delta y$.

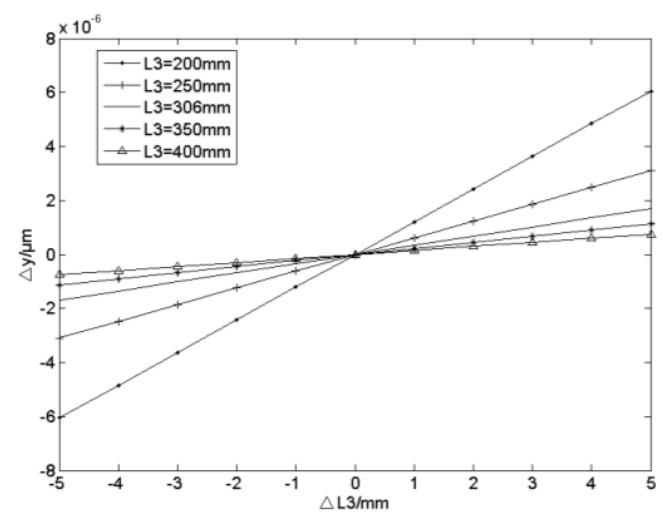

Fig. 11. The effect of error $\Delta L_{3}$ on the parallelism $\Delta y$.

\section{The Brief Summary of the Effects of Installation Errors on the Linear Parallelism}

There are 9 variables in the instrumentation to measure the horizontal and vertical parallelism and three angle errors of the linear guide pair. In order to clarify their relationship and importance, Table I is listed.

Table I show that five measuring length $x_{1}, x_{2}, x_{3}, x_{4}, x_{5}$ are important to 5 precisions, the angle of rotation $\theta_{x}, \theta_{y}, \theta_{z}$, and linear parallelism $x$. We need to make sure that the positions of sensors would keep fixed and unchanged while testing. 
TABLE I: THE RELATIONSHIP AND IMPORTANCE OF 7 VARIABLES TO 5 PRECISIONS

\begin{tabular}{|c||c|c|c|c|c|}
\hline & $\theta_{x}$ & $\theta_{y}$ & $\theta_{z}$ & $x$ & $y$ \\
\hline \hline$x_{1}$ & $\bullet$ & & & $\circ$ & $\bullet$ \\
\hline$x_{2}$ & $\bullet$ & $\bullet$ & & $\circ$ & $\circ$ \\
\hline$x_{3}$ & & $\bullet$ & & & $\bullet$ \\
\hline$x_{4}$ & & & $\bullet$ & $\bullet$ & $\circ$ \\
\hline$x_{5}$ & & & $\bullet$ & $\bullet$ & $\circ$ \\
\hline$L_{1}$ & $\circ$ & & & $\circ$ & $\circ$ \\
\hline$L_{2}$ & & $\circ$ & & & \\
\hline$L_{3}$ & & & $\circ$ & $\circ$ & $\circ$ \\
\hline$h$ & & & & $\bullet$ & \\
\hline$\bullet$ & $\bullet:$ interrelated \& critical \\
$\circ:$ interrelated
\end{tabular}

\section{CONCLUSION}

It is necessary and meaningful to test and evaluate the parallelism of guide pairs. A test instrumentation and method by using five non-contact laser displacement sensors have been put forward to measure the horizontal, vertical parallelism, $x, y$, and three rotation angles $\theta_{x}, \theta_{y}, \theta_{z}$ of the linear guide pair. The concerting formulas from the laser sensors' measurement to the parallelism of rolling linear guide pairs are derived. Because all the sensors are mounted on the testing block with some inevitably errors, the deviation of the angle of rotation $\theta_{x}, \theta_{y}, \theta_{z}$ and linear parallelism $x$ and $y$ caused by the errors are analyzed. Results show that two parallelism, $x, y$ and three rotation angles $\theta_{x}, \theta_{y}, \theta_{z}$, depend critically on five measuring length $x_{1}, x_{2}, x_{3}, x_{4}, x_{5}$. The error of them would cause great measurement error of two parallelism and three rotation angles.

\section{REFERENCES}

[1] Liu and Gao, "A method of dynamic measuring guide straightness," C.N. Patent 101329170, December 24, 2008.

[2] C. H. Liu, Y. R. Jeng, W. Y. Jywe, S. Y. Deng, and T. H. Hsu, "Automatic straightness measurement of a linear guide using a real-time straightness self-compensating scanning stage," Inst. Mech. Eng. B, J. Eng. Manuf., vol. 223, no. B9, pp. 1171-1179, September 2009.

[3] W. Li, "One automatic measuring method of linear rolling guide way," Modular Machine Tool \& Automatic Manufacturing Technique, no. 3 , pp. 34-37, 2010.

[4] L. J. Zhu, L. Li, J. H. Liua, and Z. H. Zhang, "Measuring straightness error of guide with modulated polarized light," in Proc. Fourth International Symposium on Precision Mechanical Measurements, September 2009, vol. 223, pp. 1171-1179.

[5] C. D. Hu, Y. Q. Li, J. Gao, P. Fu, H. P. Ming, and N. Ye, "Long guide straightness error measurement based on laser interference," Applied Mechanics and Materials, vol. 565, pp. 126-132, 2014.

[6] Y. Liu, Z. W. Hao, and T. T. Ren, "Inspection of large guide rail precision based on bistatic measurement method," in Proc. ISCEMP 2013, 2013, pp. 945-948.

[7] P. Majda, "Modeling of geometric errors of linear guide way and their influence on joint kinematic error in machine tools," Precision Engineering, vol. 36, no. 3, pp. 369-378, July 2012.

[8] X. Z. Yang, F. Y, Ye, J. W. Feng, and P. J. Cao, "A comprehensive precision detector of linear rolling guide," C.N. Patent 102261898A, November 30, 2011.

[9] J. Xiong, "The study of comprehensive detection principle and method of linear guide friction and movement precision," M.S. thesis, Dept. Electron. Chinese., HUST, Wuhan, China.

[10] J. L. Sun and M. H. Qian, "Analysis of the precision of linear guides motion based on GA," Journal of Huazhong Univ. of Sci. \& Tech. (Nature Science Edition), vol. 32, no. 12, December 2002.
[11] Z. S. Tao, X. H. Yin, X. F. Cai, and E. Zhang, "One comprehensive test instrument for linear motion guide," Modern Measurement and Test, no. 3, 1995.

[12] Y. Zhong, W. J. Tao, and J. Han, "Model and test study of precision loss of the roller linear guide," Modular Machine Tool \& Automatic Manufacturing Technique, no. 1, pp. 14-17, January 20, 2013.

[13] Q. Song and H. P. Wang, "A new method based on position sensitive detectors to measure four-degree-of freedom geometric errors of linear guide rails," in Proc. 2013 3rd International Conference on Mechatronics and Intelligent Materials, May 2013, vol. 706-708, pp. $1320-1323$.

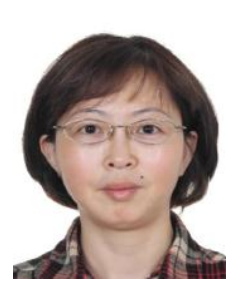

Yi Liang was born in Shaanxi, China, on August 5 , 1974. Yi Liang earned her bachelor and master degrees of mechanical engineering in Nanjing University of Science and Technology, Jiangsu province, China, in 1991 and 1998, respectively. Her major field of study is mechanical design and optimization

She has been teaching and researching in the field of mechanical engineering since 1998 in Nanjing University of Science and Technology. The courses she teaches are mechanisms and machine theory, machine elements, and mechanical optimization for the undergraduates and postgraduates. Associate professor Yi Liang focuses on the research of mechanical design and finite element analysis of machine elements. Two of her published articles are listed here: 1). "Transmission momentary efficiency based on the D'Alembert -Lagrange equation for involutes gear," Chinese Journal of Mechanical Engineering, vol. 2, pp. 272-275, Dec. 2004; 2). "Finite element analysis of Shell of mower cutter head," Journal of Machine Design, vol. 21, pp. 253-254, Jun. 2005. Now Yi is interested in the optimization design and accuracy analysis of rolling linear guide ways.

Assoc. Prof. Liang joined the membership in Nanjing Mechanical Engineering Society and its journal, Machine Building and Automation.

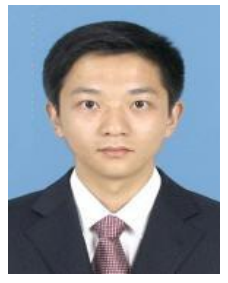

Yulin Wang was born in Nanjing, Jiangsu Province, China on December 26, 1981. In July 2003, Yulin Wang received his bachelor degree in Nanjing University of Aeronautics and Astronautics, Nanjing, Jiangsu Province, China in mechanical manufacture and automation. In July 2009, Yulin Wang received the doctor degree in Shanghai Jiao Tong University, Shanghai, China in mechanical and electronic engineering.

He has worked in Nanjing University of Science and Technology as a teacher for 5 years from December 2009. Now he is an associate professor in the Dept. of Mechanical and Electronic Engineering. From September 2013 to September 2014, he was a visiting scholar in Purdue University, Indiana, USA. His research interests are in the areas of hard turning technology, FEA, robot and manipulator, and precision measure and control technology.

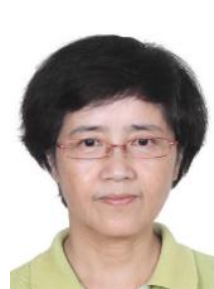

Aihua Yin was born in Shanghai, China on October 4, 1953. In July 1978, Aihua Yin graduated from Donghua University, Shanghai, China, majoring in electrical engineering and automation.

She has worked in Nanjing University of Science and Technology as a teacher for more than 30 years since August 1978. Now she is an associate professor in the Department of Mechanical and Electronic Engineering. Her research interests are in the areas of mechatronics technology, robot system design, and precision measure and control technology. Her major research projects include multifunctional mobile robot, intelligent service robot and ball screw dynamic error measurement system.

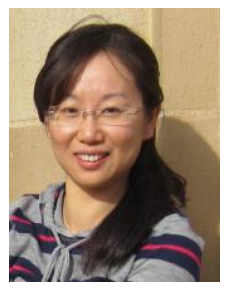

Li Zu was born in Nanjing, Jiangsu province, China on June 19, 1977. In July 1999, Li Zu received her bachelor degree in Suzhou University, Suzhou, Jiang Su Province, China in mechanical design and theory. In July $2005, \mathrm{Li} \mathrm{Zu}$ received the doctor degree in Nanjing University of Science and Technology, Nanjing, Jiangsu Province, China in mechanical and electronic engineering.

She has worked in Nanjing University of Science and Technology as a teacher for 9 years from July 2005. Now she is an associate professor in the Dept. of Mechanical Design and Automation. 
From August 2012 to January 2013, She was a visiting scholar in California State University, Northridge, USA. Now she is working on the National Natural Science Foundation Project - "Studies of the Load-bearing Performance of Precision Harmonic Gear within Abnormal Conditions and Design" and National Science and Technology Major Project - "Common Technology of Rolling Elements in the CNC Machine and Testing, Test Equipment Development Platform". There are two published articles here: 1). Li $\mathrm{Zu}$ et al., "Optimization design of the lawn mowing vehicle's blade based on aerodynamics," Advanced Materials Research, 2011, pp. 231-237.

2). Li Zu et al., "Study and design of an inchworm-1ike micro-robot walking mechanism," Lecture Notes in Electrical Engineering, vol. 123, pp. 69-78, 2011. Her research area covers mechanical design, precision drive technology and intelligent machines.

Prof. $\mathrm{Zu}$ is a member of the Association of Mechanical Engineering of Nanjing, Jiangsu Province, China.

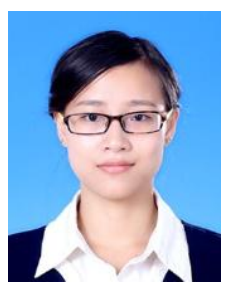

Dan Xu comes from Nanjing, Jiangsu Province, China. She was born on January 1, 1991 in Chongqing, China. Dan $\mathrm{Xu}$ is a master student in mechanical engineering, Nanjing University of Science \& Technology which located in Nanjing, China. Her research area is comprehensive performance research of linear guide.

She is working as a R\&D personnel in Zhang Jiagang Screws Precision Machinery Co. Ltd. She is studying on detection and analysis method of comprehensive properties for linear rolling guide.

Ms. Xu is a membership of research group for generic technology research of rolling components for High-grade CNC machine. 